\title{
Differential functional von Foerster equations with renewal
}

\author{
H.Leszczyński \\ Univ. Gdańsk, Wita Stwosza 57, 80-952 Gdańsk, Poland \\ Received January 31, 2008
}

Natural iterative methods converge to the exact solution of a differential-functional von Foerster-type equation which describes a single population dependent on its past time and state densities as well as on its total size. On the lateral boundary we impose a renewal condition.

Key words: iterative method, differential functional, Hale operator

PACS: $87.10 .+e, 82.39 .-k, 82.39 . R k$

\section{Introduction}

The paper is dedicated to the memory of Professor A. Lasota. Von Foerster and Volterra-Lotka equations arise in biology, medicine and chemistry, $[1,7,11,12,14]$. The independent variables $x_{j}$ and the unknown function $u$ stand for certain features and densities, respectively. It follows from this natural interpretation that $x_{j} \geqslant 0$ and $u \geqslant 0$. Von Foerster model is essentially nonlocal, because it contains the total size of population $\int u(t, x) \mathrm{d} x$.

Existence results for certain von Foerster type problems have been established by means of the Banach contraction principle, the Schauder fixed point theorem or iterative methods, see [2] and [3-5]. Nonlocal terms always cause huge problems. Satisfactory conditions for convergence of iterative methods were provided in [9], where (for the sake of simplicity) some boundary data were prescribed. This forced additional restrictions on the (tangential!) flow of bicharacteristics near the lateral boundary.

In the present paper we generalize the $L^{\infty} \cap L^{1}$-convergence results of [9] to the case of renewal boundary conditions with natural assumptions on the flow of bicharacteristics. An associate result to [9] on fast convergent quasi-linearization methods has been published in [8]. The renewal condition of the form

$$
u(t, 0)=\int_{0}^{\infty} k(t, x, y) u(t, x) \mathrm{d} x
$$

is interpreted as giving birth to young individuals at the age 0 by mature individuals $(0<x<+\infty)$. In A. Lasota's investigations there were included some delay effects like $\int u(t-r, x) \mathrm{d} x$. Such cases are also available using the method of the present paper. Investigations of finite difference schemes on large meshes with strongly nonlocal functionals were analyzed in [15]. Since the renewal case is complex, we arrive at its reduction to the one with given data on the lateral boundary. In fact, the unknown renewal part is expressed by a Green function. This approach can be extended to many species models. There is also a difference between the renewal case and [9]: the renewal involves such coupling of the unknown function that excludes monotone iterations.

\subsection{Formulation of the differential problem.}

Let $\tau=\left(\tau_{1}, \ldots, \tau_{n}\right) \in \mathbf{R}_{+}^{n}, \tau_{0}>0$, where $\mathbf{R}_{+}:=[0,+\infty)$. Define

$$
B=\left[-\tau_{0}, 0\right] \times[-\tau, \tau], \text { where }[-\tau, \tau]=\left[-\tau_{1}, \tau_{1}\right] \times \cdots \times\left[-\tau_{n}, \tau_{n}\right]
$$


and

$E_{0}=\left[-\tau_{0}, 0\right] \times \mathbf{R}^{n}, \quad E=[0, a] \times \mathbf{R}^{n}, \quad(a>0), \quad \partial E=\left\{(t, x) \in E: x_{1} \cdots x_{n}=0\right\}$.

For each function $w$ defined on $\left[-\tau_{0}, a\right]$, we have the Hale functional $w_{t}$ (see [6]), which is the function defined on $\left[-\tau_{0}, 0\right]$ by

$$
w_{t}(s)=w(t+s), \quad\left(s \in\left[-\tau_{0}, 0\right]\right) .
$$

For each function $u$ defined on $E_{0} \cup E$, we similarly write a Hale-type functional $u_{(t, x)}$, defined on $B$ by

$$
u_{(t, x)}(s, y)=u(t+s, x+y) \quad \text { for }(s, y) \in B .
$$

Let $\Omega_{0}=E \times C\left(\left[-\tau_{0}, a\right], \mathbf{R}_{+}\right)$and $\Omega=E \times C\left(B, \mathbf{R}_{+}\right) \times C\left(\left[-\tau_{0}, a\right], \mathbf{R}_{+}\right)$. Take $v: E_{0} \rightarrow \mathbf{R}_{+}$ and

$$
c_{j}: \Omega_{0} \rightarrow \mathbf{R}, \quad \lambda: \Omega \rightarrow \mathbf{R} \quad(j=1, \ldots, n) .
$$

Consider the differential-functional equation

$$
\frac{\partial u}{\partial t}+\sum_{j=1}^{n} c_{j}\left(t, x, z[u]_{t}\right) \frac{\partial u}{\partial x_{j}}=u(t, x) \lambda\left(t, x, u_{(t, x)}, z[u]_{t}\right),
$$

where

$$
z[u](t):=\int_{\mathbf{R}_{+}^{n}} u(t, y) \mathrm{d} y, \quad t \in\left[-\tau_{0}, a\right],
$$

with the initial conditions

$$
u(t, x)=v(t, x), \quad(t, x) \in E_{0}, \quad x=\left(x_{1}, \ldots, x_{n}\right) \in \mathbf{R}_{+}^{n},
$$

and with the renewal condition

$$
u(t, x)=\int_{\mathbf{R}_{+}^{n}} k(t, x, y) u(t, y) \mathrm{d} y, \quad(t, x) \in \partial E, \quad y \in \mathbf{R}_{+}^{n},
$$

where $k: \partial E \times \mathbf{R}_{+}^{n} \rightarrow \mathbf{R}_{+}^{n}$. We are looking for Caratheodory's solutions to (1)-(4), see [2] and [10]. The functional dependence includes a possible delayed and integral dependence of the Volterra type. The Hale functional $z[u]_{t}$ takes into consideration the whole population within the time interval $\left[t-\tau_{0}, t\right]$, whereas the Hale-type functional $u_{(t, x)}$ shows the dependence on the density $u$ locally in a left neighbourhood of $(t, x)$. For simplicity we assume in the paper that $n=1$. Notice that it is possible to extend the result to the case $n>1$ with quite technical multiple integrals on the lateral boundary.

\section{Bicharacteristics}

First, for a given function $z \in C\left(\left[-\tau_{0}, a\right], \mathbf{R}_{+}\right)$, consider the bicharacteristic equations for problem (1), (3):

$$
\eta^{\prime}(s)=c\left(s, \eta(s), z_{s}\right), \quad \eta(t)=x .
$$

Denote by $\eta=\eta[z](\cdot ; t, x)=\left(\eta_{1}[z](\cdot ; t, x), \ldots, \eta_{n}[z](\cdot ; t, x)\right)$ the bicharacteristic curve passing through $(t, x) \in E$, i.e., the solution to problem (5). We consider its maximal (left) existence domain to be an interval $[\alpha(t, x), t]$, where $\alpha(t, x)=0$ or $0<\alpha(t, x) \leqslant t$. This alternative splits $E$ into two parts $E_{0}[z]$ and $E_{+}[z]$. Next, we consider the following equation

$$
\frac{\mathrm{d}}{\mathrm{d} s} u(s, \eta[z](s ; t, x))=u(s, \eta[z](s ; t, x)) \lambda\left(s, \eta[z](s ; t, x), u_{(s, \eta[z](s ; t, x))}, z_{s}\right)
$$

with the initial condition

$$
u(0, \eta[z](0 ; t, x))=v(0, \eta[z](0 ; t, x)) \text { for }(t, x) \in E_{0}[z]
$$


and (with the brief notation $\alpha=\alpha(t, x)$ )

$$
u(\alpha, \eta[z](\alpha ; t, x))=\tilde{v}(\alpha, \eta[z](\alpha ; t, x)) \quad \text { for } \quad(t, x) \in E_{+}[z] .
$$

In the latter equation (6) the existence of a suitable extension $\tilde{v}$ of $v$ to the lateral boundary is complicated. We discuss this topic later on.

For any given function $z \in C\left(\left[-\tau_{0}, a\right], \mathbf{R}_{+}\right)$, a solution of equation (6) along bicharacteristics (5) is a solution of (1). The initial conditions (3) and (7) or (8) correspond to each other.

Assume that:

(V0) $v \in C B\left(E_{0}, \mathbf{R}_{+}\right)$(non-negative, bounded and continuous function);

(V1) $z[v] \in C\left(\left[-\tau_{0}, 0\right], \mathbf{R}_{+}\right)$, where

$$
z[v](t)=\int_{\mathbf{R}^{n}} v(t, x) \mathrm{d} x
$$

(V2) the function $v$ satisfies the Lipschitz condition

$$
|v(t, x)-v(t, \bar{x})| \leqslant L_{v}\|x-\bar{x}\| \quad \text { on } \quad E_{0}
$$

with some constant $L_{v}>0$;

(C0) $c_{j}: \Omega_{0} \rightarrow \mathbf{R}_{+}$are positive, continuous and

$$
\|c(t, x, q)-c(t, \bar{x}, \bar{q})\| \leqslant L_{c}\|x-\bar{x}\|+L_{c}^{*}\|q-\bar{q}\| .
$$

A continuous function $\sigma:[0, a] \times \mathbf{R}_{+} \rightarrow \mathbf{R}_{+}$is said to be a Perron comparison function if $\sigma(t, 0) \equiv 0$ and the differential problem $y^{\prime}=\sigma(t, y), y(0)=0$ has the only zero solution. We call it uniform if $\sigma$, multiplied by any positive constant, is also a Perron comparison function. We call it monotone if $\sigma$ is non-decreasing in the second variable.

$(\Lambda \mathbf{0}) \lambda: \Omega \rightarrow \mathbf{R}$ is continuous in $(t, x, w, q)$ and

$$
|\lambda(t, x, w, q)-\lambda(t, \bar{x}, \bar{w}, \bar{q})| \leqslant M_{\lambda} \sigma(t,\|x-\bar{x}\|+\|w-\bar{w}\|+\|q-\bar{q}\|),
$$

where $\sigma:[0, a] \times \mathbf{R}_{+} \rightarrow \mathbf{R}_{+}$is a monotone, uniform Perron comparison function;

( $\Lambda \mathbf{1})$ there exists a function $L_{\lambda} \in L^{1}\left([0, a], \mathbf{R}_{+}\right)$, such that

$$
\lambda(t, x, w, q) \leqslant L_{\lambda}(t)
$$

for $(t, x) \in E, w \in C\left(B, \mathbf{R}_{+}^{m}\right), q \in C\left(\left[-\tau_{0}, a\right], \mathbf{R}_{+}^{m}\right)$.

Denote

$$
W(t, x, w, q)=\lambda(t, x, w, q)+\operatorname{tr} \partial_{x} c(t, x, q)
$$

for $(t, x) \in E, w \in C\left(B, \mathbf{R}_{+}\right), q \in C\left(\left[-\tau_{0}, a\right], \mathbf{R}_{+}\right)$, where $\operatorname{tr} \partial_{x} c$ stands for the trace of the matrix $\partial_{x} c=\left[\partial_{x_{k}} c_{j}\right]_{j, k=1, \ldots, n}$.

(W0) There exists $M_{W} \in \mathbf{R}_{+}$, such that

$$
|W(t, x, w, q)-W(t, \bar{x}, \bar{w}, \bar{q})| \leqslant M_{W} \sigma(t,\|x-\bar{x}\|+\|w-\bar{w}\|+\|q-\bar{q}\|),
$$

where $\sigma:[0, a] \times \mathbf{R}_{+} \rightarrow \mathbf{R}_{+}$is a monotone, uniform Perron comparison function.

(W1) There exists a function $L_{W} \in L^{1}\left([0, a], \mathbf{R}_{+}\right)$, such that

$$
W(t, x, w, q) \leqslant L_{W}(t)
$$

for $(t, x) \in E, w \in C\left(B, \mathbf{R}_{+}\right), q \in C\left(\left[-\tau_{0}, a\right], \mathbf{R}_{+}\right)$.

(K0) The kernel $k: \partial E \times \mathbf{R}_{+}^{n} \rightarrow \mathbf{R}_{+}^{n}$ is bounded and continuous, and $a \cdot\|k\|_{\infty} \cdot\|c\|<1$.

The latter condition states that the length of the interval $[0, a]$ is sufficiently small. This assumption is superfluous if $k(t, x, y)=0$. 
Lemma 2.1 If the conditions (V0), ( $(1)$, (K0) are satisfied, then any solution u of equation (6) has the estimate

$$
0 \leqslant u(t, x) \leqslant\|v\|_{\infty} \exp \left(\int_{0}^{t} L_{\lambda}(s) \mathrm{d} s\right) \quad \text { on } \quad E_{0}[z]
$$

and

$$
0 \leqslant u(t, x) \leqslant\|v\|_{\infty} \exp \left(\int_{0}^{t}\left(L_{\lambda}(s)+L_{W}(s)\right) \mathrm{d} s\right) \cdot \frac{\|k\|_{\infty}}{1-a \cdot\|k\|_{\infty} \cdot\|c\|_{\infty}} \quad \text { on } E_{+}[z] .
$$

Proof. The first inequality is standard. The second one will be explained in the following subsection.

\subsection{The fixed point equation.}

Let

$$
\begin{aligned}
Z(t)= & \max _{-\tau_{0} \leqslant s \leqslant 0}\|v(s, \cdot)\|_{1} \exp \left(\int_{0}^{t} L_{W}(s) \mathrm{d} s\right) \\
& +t \cdot\|c\|_{\infty} \cdot\|v\|_{\infty} \exp \left(\int_{0}^{t}\left(L_{\lambda}(s)+L_{W}(s)\right) \mathrm{d} s\right) \cdot \frac{\|k\|_{\infty}}{1-a \cdot\|k\|_{\infty} \cdot\|c\|_{\infty}}
\end{aligned}
$$

where we put $L_{W}(s)=0$ for $s \in\left[-\tau_{0}, 0\right]$, and

$$
\mathcal{Z}=\left\{z \in C\left(\left[-\tau_{0}, a\right], \mathbf{R}_{+}\right): z(t) \leqslant Z(t)\right\} .
$$

Consider the operator $\mathcal{T}: \mathcal{Z} \rightarrow \mathcal{Z}$ given by the formula

$$
\mathcal{T}[z](t)=\int_{\mathbf{R}_{+}^{n}} u[z](t, x) \mathrm{d} x \quad \text { for } \quad \mathrm{t} \geqslant 0,
$$

where $u=u[z] \in C^{1}\left(B, \mathbf{R}_{+}\right)$is the solution of (6)-(7) with the initial condition $u[z](t, x)=v(t, x)$ on $E_{0}$. The function $u=u[z]$ has the following integral representation

$$
u[z](t, x)=v(\alpha, \eta(\alpha)) \exp \left(\int_{\alpha}^{t} \lambda\left(s, \eta(s), u_{(s, \eta(s))}, z_{s}\right) \mathrm{d} s\right),
$$

where $\eta(s)=\eta[z](s ; t, x)$ and $\alpha=\alpha(t, x)$ ( $\alpha$ depends on $z$ ). By Lemma 2.1, we write (11) in the following way

$$
\mathcal{T}[z](t)=\int_{\mathbf{R}_{+}^{n}} v(\alpha, \eta(\alpha)) \exp \left(\int_{\alpha}^{t} \lambda\left(s, \eta(s), u_{(s, \eta(s))}, z_{s}\right) \mathrm{d} s\right) \mathrm{d} x
$$

for $t \geqslant 0$. The bicharacteristics admit the group property:

$$
y=\eta[z](0 ; t, x) \Longleftrightarrow \eta[z](s ; t, x)=\eta[z](s ; 0, y),
$$

that is: any bicharacteristic curve passing through the points $(0, y)$ and $(t, x)$ has the same value at $s \in[0, a]$.

If we change the variables $y=\eta[z](0 ; t, x)$, then, by the Liouville theorem, the Jacobian $J=\operatorname{det}\left[\frac{\partial c}{\partial x}\right]$ is given for $\alpha=\alpha(t, x)$ by the formula

$$
J(\alpha ; t, x)=\exp \left(-\int_{\alpha}^{t} \operatorname{tr} \partial_{x} c\left(s, \eta_{i}[z](s ; 0, y), z_{s}\right) \mathrm{d} s\right)
$$


Hence (13) can be written in the form

$$
\begin{aligned}
\mathcal{T}[z](t)= & \int_{\mathbf{R}_{+}^{n}} v(0, y) \exp \left(\int_{0}^{t} W\left(s, \eta(s), u_{(s, \eta(s))}, z_{s}\right) \mathrm{d} s\right) \mathrm{d} y \\
& +\int_{S_{t}} u(\alpha, \eta(\alpha ; t, x)) \exp \left(\int_{\alpha}^{t} \lambda\left(s, \eta(s), u_{(s, \eta(s))}, z_{s}\right) \mathrm{d} s\right) \mathrm{d} x,
\end{aligned}
$$

where $\eta(s)=\eta[z](s ; 0, y), \alpha=\alpha(t, x)$ and $S_{t}$ is the set of $x \in \mathbf{R}_{+}^{n}$ such that $\alpha(t, x)>0$.

Lemma 2.2 If the conditions (V0), (V1), (W1), (K0) are satisfied, then

$$
0 \leqslant \mathcal{T}[z](t) \leqslant Z(t)<+\infty \quad \text { for } \quad t \in[0, a]
$$

where $Z$ is given by (9).

Proof. This assertion follows directly from (14) and Assumptions (V0), (V1) and (W1).

The respective fixed point equation for bicharaceristics $\eta=\eta[z]$ has the form

$$
\eta(s ; t, x)=x-\int_{s}^{t} c\left(\zeta, \eta(\zeta ; t, x), z_{\zeta}\right) \mathrm{d} \zeta .
$$

Lemma 2.3 If Assumption (CO) is satisfied and $z, \bar{z} \in \mathcal{Z}$, then

$$
\|\eta[z](s ; t, x)-\eta[\bar{z}](s ; t, x)\| \leqslant \int_{s}^{t} L_{c}^{*}\left\|z_{\zeta}-\bar{z}_{\zeta}\right\| \mathrm{e}^{L_{c}(\zeta-s)} \mathrm{d} \zeta .
$$

Lemma 2.4 Under the Assumptions (V0), (V1), (C0), (KO), for each $z \in \mathcal{Z}$, there exists the unique continuous function $\tilde{v}: \partial E \rightarrow \mathbf{R}_{+}$which satisfies the renewal condition.

Proof. The renewal condition (4) for $(t, x) \in \partial E$ can be rewritten as follows

$$
u(t, x)=\int_{\mathbf{R}_{+}^{n} \backslash S_{t}} k(t, x, y) u(t, y)+\int_{S_{t}} k(t, x, y) u(t, y) \mathrm{d} y .
$$

The first term is a bounded operator of $v$, see (12). From (12), the second term is equal to

$$
\int_{S_{t}} k(t, x, y) v(\alpha, \eta(\alpha)) \exp \left(\int_{\alpha}^{t} \lambda\left(s, \eta(s), u_{(s, \eta(s))}, z_{s}\right) \mathrm{d} s\right) \mathrm{d} y,
$$

where $\eta(s)=\eta[z](s ; t, y)$ and $\alpha=\alpha(t, y)$. By (K0) the second term is small (has the norm less than 1).

The above lemma explains the estimate of Lemma 2.1. The next statement is crucial in our paper.

Lemma 2.5 Under all previous assumptions, any solution $z$ of the fixed point equation for (14) has the representation

$$
z(t)=\int_{R_{+}^{n}} v(0, y) G(t, y ; z) \mathrm{d} y
$$

where the Green function $G$ has the same estimate as the first kernel in (14), multiplied by some constant.

Proof. The assertion follows from a Neumann series expansion for $u$ aided by the renewal condition. 


\section{The iterative method.}

Define the iterative method by $z^{(k+1)}=\mathcal{T}\left[z^{(k)}\right]$ with an arbitrary function $z^{(0)} \in \mathcal{Z}$, where the class $\mathcal{Z}$ is defined by (10). We prove its uniform convergence under natural assumptions on the given functions. The algorithm splits into three stages:

1. finding bicharacteristics $\eta^{(k)}=\eta\left[z^{(k)}\right]$, given by (15),

2. finding $u^{(k)}=u\left[z^{(k)}\right]$ as a solution of $(12)$,

3. calculating $z^{(k+1)}=\mathcal{T}\left[z^{(k)}\right]$ by means of (13) or (14). In this way there are given the integral equations

$$
\begin{aligned}
\eta^{(k)}(s ; t, x)= & x-\int_{s}^{t} c\left(\zeta, \eta^{(k)}(\zeta ; t, x), z_{\zeta}^{(k)}\right) \mathrm{d} \zeta \\
u^{(k)}(t, x)= & v\left(\alpha, \eta^{(k)}(\alpha ; t, x)\right) \exp \left(\int_{\alpha}^{t} \lambda\left(Q^{(k)}(s)\right) \mathrm{d} s\right) \\
z^{(k+1)}(t)= & \int_{\mathbf{R}^{n}} v(0, y) \exp \left(\int_{0}^{t} W\left(R^{(k)}(s)\right) \mathrm{d} s\right) \mathrm{d} y \\
& +\int_{S_{t}} u\left(\alpha, \eta^{(k)}(\alpha ; t, x)\right) \exp \left(\int_{\alpha}^{t} \lambda\left(Q^{(k)}(s)\right) \mathrm{d} s\right)
\end{aligned}
$$

where $\alpha=\alpha(t, x)$ (depends on $\left.z^{(k)}\right)$ and

$$
Q^{(k)}(s)=\left(s, \eta^{(k)}(s ; t, x), u_{\left(s, \eta^{(k)}(s ; t, x)\right)}^{(k)}, z_{s}^{(k)}\right), \quad R^{(k)}(s)=\left(s, \eta^{(k)}(s ; 0, y), u_{\left(s, \eta^{(k)}(s ; 0, y)\right)}^{(k)}, z_{s}^{(k)}\right)
$$

Theorem 3.1 If $z^{(0)} \in \mathcal{Z}$ and Assumptions $(V 0)-(V 2),(C 0),(\Lambda 0),(\Lambda 1),(W 0),(W 1),(K 0)$ are satisfied and there are $K \in \mathbf{R}_{+}, \theta \in(0,1]$ such that

$$
\sigma(t, r) \leqslant K t^{\theta-1} p r^{1-1 / p} \quad \text { for } p \geqslant 2,
$$

then the iterative method $z^{(k+1)}=\mathcal{T}\left[z^{(k)}\right]$ is well defined in $\mathcal{Z}$ and uniformly converges to the unique fixed point $z=\mathcal{T}[z]$, locally, that is: on a sufficiently small $[0, a]$.

Remark 3.1 The technical condition (16) is fulfilled in the Lipschitz case $(\sigma(t, r)=L r)$ as well as the simplest nonlinear Perron comparison functions such as $\sigma(t, r)=L r \ln (1+1 / r)$. Its formulation also includes weak singularities, e.g. $\sigma(t, r)=t^{-1 / 2} L r$ and $\sigma(t, r)=t^{-1 / 2} L r \ln (1+1 / r)$.

Proof. (of Theorem 3.1) Denote

$$
\Delta z^{(k)}=z^{(k+1)}-z^{(k)}, \Delta \eta^{(k)}=\eta^{(k+1)}-\eta^{(k)}, \Delta u^{(k)}=u^{(k+1)}-u^{(k)} .
$$

Then we have the estimates

$$
\begin{aligned}
\left\|\Delta \eta^{(k)}(s ; t, x)\right\| \leqslant & \int_{s}^{t} L_{c}^{*}\left\|\Delta z_{\zeta}^{(k)}\right\| \mathrm{e}^{L_{c}(\zeta-s)} \mathrm{d} \zeta \\
\left|\Delta u^{(k)}(t, x)\right| \leqslant & C\left\|\Delta \eta^{(k)}(0 ; t, x)\right\| \exp \left(\int_{0}^{t} L_{\lambda}(s) \mathrm{d} s\right) \\
& +C \exp \left(\int_{0}^{t} L_{\lambda}(s) \mathrm{d} s\right) \int_{0}^{t} M_{\lambda} \sigma\left(s, P^{(k)}(s ; t, x)\right) \mathrm{d} s \\
\left|\Delta z^{(k+1)}(t)\right| \leqslant & C \int_{0}^{t} M_{W} \sigma\left(s, P^{(k)}(s ; t, x)\right) \mathrm{d} s
\end{aligned}
$$

where $P^{(k)}(s ; t, x)=\left\|\Delta \eta^{(k)}(s ; t, x)\right\|+\left\|\Delta u^{(k)}\right\|_{s}+\left\|\Delta z^{(k)}\right\|_{s}$. In the above estimate the constant $C$ is generic (depending on the data). 
Denote $\hat{L}_{\lambda}=\int_{0}^{a} L_{\lambda}(s) \mathrm{d} s$ and

$$
\Psi^{(k)}(s, t)=\bar{\psi}^{(k)}(s)+\psi^{(k)}(s)+\int_{s}^{t} L_{c}^{*} \mathrm{e}^{L_{c} a} \psi^{(k)}(\zeta) \mathrm{d} \zeta .
$$

Consider the comparison equations

$$
\begin{aligned}
\bar{\psi}^{(k)}(t) & =C \int_{0}^{t} L_{c}^{*} \mathrm{e}^{L_{c} a+\hat{L}_{\lambda}} \psi^{(k)}(s) \mathrm{d} s+C e^{\hat{L}_{\lambda}} \int_{0}^{t} M_{\lambda} \sigma\left(s, \Psi^{(k)}(s, t)\right) \mathrm{d} s, \\
\psi^{(k+1)}(t) & =C \int_{0}^{t} M_{W} \sigma\left(s, \Psi^{(k)}(s, t)\right) \mathrm{d} s
\end{aligned}
$$

with $\psi^{(0)}(t)=Z(t)$ and

$$
\begin{aligned}
\bar{\psi}^{(0)}(t) & =C \exp \left(\int_{0}^{t} L_{W}(s) \mathrm{d} s\right)+C \int_{0}^{t} L_{c}^{*} \mathrm{e}^{L_{c} a+\hat{L}_{\lambda}} Z(s) \mathrm{d} s \\
& +C \mathrm{e}^{\hat{L}_{\lambda}} \int_{0}^{t} M_{\lambda} \sigma\left(s, \bar{\psi}^{(0)}(s)+Z(s)+\int_{s}^{t} L_{c}^{*} \mathrm{e}^{L_{c} a} Z(\zeta) \mathrm{d} \zeta\right) \mathrm{d} s .
\end{aligned}
$$

The remaining part of the proof is split into several auxiliary lemmas.

Lemma 3.1 Under the assumptions of Theorem 3.1 there is $a_{0} \in(0, a]$ such that $\left|\Delta u^{(k)}(t, x)\right| \leqslant$ $\bar{\psi}^{(k)}(t),\left|\Delta z^{(k)}(t)\right| \leqslant \psi^{(k)}(t)$,

$$
\left\|\Delta \eta^{(k)}(s ; t, x)\right\| \leqslant \int_{s}^{t} L_{c}^{*} \mathrm{e}^{L_{c} a} \psi^{(k)}(\zeta) \mathrm{d} \zeta
$$

on $\left[0, a_{0}\right] \times \mathbf{R}_{+}$, and the sequences $\left\{\psi^{(k)}\right\},\left\{\bar{\psi}^{(k)}\right\}$ are non-decreasing in $k$.

Lemma 3.2 Under the assumptions of Theorem 3.1 the estimate

$$
\int_{0}^{t} \sigma\left(s, A s^{l}+B t^{l+1}\right) \mathrm{d} s \leqslant t^{l+\theta-l / p} p K \theta^{-1}\left[\frac{A}{\theta+l}+\frac{B a}{\theta}\right]^{1-1 / p}
$$

holds.

Lemma 3.3 Under the assumptions of Theorem 3.1 the sequences $\left\{\psi^{(k)}\right\}$ and $\left\{\bar{\psi}^{(k)}\right\}$ tend uniformly to 0 as $k \rightarrow+\infty$.

Proof. With some constants $M, M^{*}$ and $c_{a}$, dependent on the data, the equation

$$
\hat{\psi}(t)=M \int_{0}^{t} \hat{\psi}(s) \mathrm{d} s+M^{*} \int_{0}^{t} \sigma\left(s, \hat{\psi}(s)+c_{a} \int_{s}^{t} \hat{\psi}(\zeta) \mathrm{d} \zeta\right) \mathrm{d} s
$$

describes a comparison function $\hat{\psi}$ with respect to $\psi+\bar{\psi}$, where

$$
\psi(t)=\lim _{k \rightarrow \infty} \psi^{(k)}(t), \quad \bar{\psi}(t)=\lim _{k \rightarrow \infty} \bar{\psi}^{(k)}(t) .
$$

One can prove by induction on $k=0,1, \ldots$ that $\hat{\psi}(t) \leqslant \hat{C}_{k} t^{\theta / 2}$ and $\hat{C}_{k} a^{\theta / 2} \rightarrow 0$ as $k \rightarrow+\infty$, provided that the interval $[0, a]$ is sufficiently small. Take an arbitrary $\hat{C}_{0}$ which estimates $\hat{\psi}(t)$. Applying Lemma 3.2 with $p=2$ to (20), we get

$$
\hat{\psi}(t) \leqslant M t \hat{C}_{0}+M^{*} t^{\theta} 2 K \theta^{-1}\left[\frac{\hat{C}_{0}\left(1+c_{a} a\right)}{\theta}\right]^{1 / 2} \leqslant t^{\theta / 2} \hat{C}_{1},
$$


where

$$
\hat{C}_{1}=M a^{1-\theta / 2} \hat{C}_{0}+a^{\theta / 2} 2 K \theta^{-1}\left[\frac{\hat{C}_{0}\left(1+c_{a} a\right)}{\theta}\right]^{1 / 2} .
$$

Suppose that the desired estimate holds for some $k \geqslant 1$. Applying Lemma 3.2 with $p=2 k$ to (20), we get

$$
\hat{\psi}(t) \leqslant M \frac{t^{1+k \theta / 2}}{1+k \theta / 2} \hat{C}_{k}+M^{*} t^{(k+1) \theta / 2} 2 K \theta^{-1}\left[\frac{\hat{C}_{k}}{\theta+k \theta / 2}+\frac{c_{a} \hat{C}_{k}}{\theta(1+k \theta / 2)}\right]^{1-1 /(2 k)}
$$

hence $\hat{\psi}(t) \leqslant t^{(k+1) \theta / 2} \hat{C}_{k+1}$, where

$$
\hat{C}_{k+1}=M \hat{C}_{k} \frac{a^{1-\theta / 2}}{1+k \theta / 2}+M^{*} 2 K \theta^{-1}\left[\frac{\hat{C}_{k}}{\theta+k \theta / 2}+\frac{c_{a} \hat{C}_{k}}{\theta(1+k \theta / 2)}\right]^{1-1 /(2 k)} .
$$

The constants $\hat{C}_{k}$ have an upper estimate of the form $A Q^{k}$, thus $\hat{\psi}(t) \equiv 0$ in a neighbourhood of 0 (because $\hat{\psi}(t) \leqslant A Q^{k} t^{k \theta / 2}$ ).

Lemma 3.4 Under the assumptions of Theorem 3.1 the sequences $\left\{z^{(k)}\right\},\left\{u^{(k)}\right\},\left\{\eta^{(k)}\right\}$ tend uniformly to $z, u[z], \eta[z]$ such that $z=\mathcal{T}[z]$.

Proof. We intend to find the following estimates

$$
\psi^{(k)}(t) \leqslant C_{k} t^{l_{k}}, \quad \bar{\psi}^{(k)}(t) \leqslant \bar{C}_{k} t^{l_{k}},
$$

where the series $\sum_{k} C_{k} t^{l_{k}}$ is convergent in a neighbourhood of 0 . The assertion is seen if we replace the comparison equations (17)-(18) by the inequalities

$$
\begin{gathered}
\bar{C}_{k} t^{l_{k}} \geqslant C \int_{0}^{t} L_{c}^{*} \mathrm{e}^{L_{c} a+\hat{L}_{\lambda}} C_{k} s^{l_{k}} \mathrm{~d} s+C \mathrm{e}^{\hat{L}_{\lambda}} \int_{0}^{t} M_{\lambda} \sigma\left(s,\left(C_{k}+\bar{C}_{k}\right) s^{l_{k}}+L_{c}^{*} \mathrm{e}^{L_{c} a} C_{k} t^{l_{k}+1} /\left(l_{k}+1\right)\right) \mathrm{d} s \\
C_{k+1} t^{l_{k+1}} \geqslant C \int_{0}^{t} M_{W} \sigma\left(s,\left(C_{k}+\bar{C}_{k}\right) s^{l_{k}}+L_{c}^{*} \mathrm{e}^{L_{c} a} C_{k} t^{l_{k}+1} /\left(l_{k}+1\right)\right) \mathrm{d} s
\end{gathered}
$$

with suitable $C_{0} t^{l_{0}} \geqslant Z(a)$ and with some

$$
\bar{C}_{0} \geqslant a C L_{c}^{*} \mathrm{e}^{L_{c} a+\hat{L}_{\lambda}} Z(a)+C \mathrm{e}^{\hat{L}_{\lambda}} M_{\lambda} \int_{0}^{a} \sigma\left(s, \bar{C}_{0}+Z(a)+a L_{c}^{*} \mathrm{e}^{L_{c} a} Z(a)\right) \mathrm{d} s .
$$

If we put

$$
l_{0}=0, \quad p_{0}=2 / \theta, \quad l_{k}=k \theta / 2, \quad p_{k}=4 k \quad \text { for } k=1,2, \ldots
$$

and exploit Lemma 3.2, then $C_{k}, \bar{C}_{k}$ can be defined as making the series $\sum_{k} C_{k} t^{l_{k}}$ convergent, hence the series $\psi^{(0)}+\psi^{(2)}+\ldots$ uniformly converges, and $z^{(k)}$ has a limit, which is continuous.

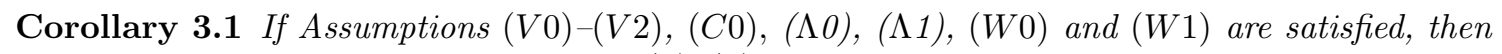
there exists a unique solution of problem (1)-(3), locally with respect to $t$.

\subsection{The Lipschitz case.}

Suppose that Assumptions $(V 0)-(V 2),(C 0),(\Lambda 1)$ and $(W 1),(\mathrm{K} 0)$ formulated in Section 2, are valid. We modify some assumptions on the functions $\lambda$ and $W$ as follows:

$(\tilde{\Lambda} \mathbf{0}) \lambda: \Omega \rightarrow \mathbf{R}$ is continuous in $(t, x, w, q)$ and there exists a function $L \in L^{1}\left([0, a], \mathbf{R}_{+}\right)$, such that

$$
|\lambda(t, x, w, q)-\lambda(t, \bar{x}, \bar{w}, \bar{q})| \leqslant L(t)(\|x-\bar{x}\|+\|w-\bar{w}\|+\|q-\bar{q}\|)
$$


( $\tilde{W} \mathbf{0}) W: \Omega \rightarrow \mathbf{R}$ and there exists a function $L \in L^{1}\left([0, a], \mathbf{R}_{+}\right)$, such that

$$
|W(t, x, w, q)-W(t, \bar{x}, \bar{w}, \bar{q})| \leqslant L(t)(\|x-\bar{x}\|+\|w-\bar{w}\|+\|q-\bar{q}\|) .
$$

Using the same notation as in the proof of Theorem 3.1, we have the estimates of the form

$$
\psi^{(k)}(t) \leqslant \frac{Q^{k+1}\left(\int_{0}^{t} \Delta(s) \mathrm{d} s\right)^{k}}{k !}, \quad t \in[0, a],
$$

where $Q$ is a generic constant. Hence the sequences $\left\{\psi^{(k)}\right\}$ and $\left\{\bar{\psi}^{(k)}\right\}$ tend uniformly to 0 as $k \rightarrow+\infty$.

\section{References}

1. Brauer F., Castillo-Chávez C. Mathematical Models in Population Biology and Epidemiology. SpringerVerlag, New York, 2001.

2. Dawidowicz A.L. Existence and uniques of solution of generalized von Foerster integro-differential equation with multidimensional space of characteristics of maturity. Bull. Acad. Polon. Sci. Math., 1990, 38, 1-12.

3. Von Foerster H. Some remarks on changing populations - In: The Kinetics of Celural Proliferation, Grune and Stratton, New York, 1959.

4. Gurtin M.E. A system of equations for age-dependent population diffusion. J. Theor. Biol., 1973, 40, 389-392.

5. Gurtin M.E., McCamy R. Non-linear age-dependend Population Dynamics. Arch. Rat. Mech. Anal., 1974, 54, 281-300.

6. Hale J.K., Lunel S.V. Introduction to Functional Differential Equations. Springer-Verlag, New York, Applied Mathematical Sciences Vol. 99, 1993.

7. Keyfitz N. Introduction to the Mathematics of Population. Addison-Wesley, Reading, 1968.

8. Leszczyński H. Fast convergent iterative methods for some problems of mathematical biology. Proceedings of the Conference Differential \& Difference Equations and Applications, Hindavi Publishing Corporation, 2006, 661-666.

9. Leszczyński H., Zwierkowski P. Iterative methods for generalized von Foerster equations with functional dependence, J. Inequal. Appl., 2007, Art. ID 12324, 14.

10. Leszczyński H., Zwierkowski P. Existence of solutions to generalized von Foerster equations with functional dependence. Ann. Polon. Math., 2004, 83, 3, 201-210.

11. Lotka A.J. Elements of Physical Biology. Wiliams and Wilkins, Baltimore 1925, republished as Elements of Mathematical Biology. Dover, New York, 1956.

12. Nakhushev A.M. Equations of Mathematical Biology. Vysshaya Shkola, Moscow, 1995 (in Russian).

13. Szarski J. Differential inequalities. PWN, Warszawa, 1965.

14. Verhulst P.F. Recherches mathématiques sur la loi d'accroissement de la population. Mém. Acad. Roy. Bruxelles 18, 1.

15. Zwierkowski P. Stability of forward-backward finite difference schemes for certain problems in biology. Comment. Math., 2005, 45, 191-203. 


\section{Диференціальні функціональні рівняння Фон Фьорстера 3 відновленням}

\section{Г.Лещиньскі}

Університет Ґданська, Ґданськ, Польща

Отримано 31 січня 2008 р.

Природні ітеративні методи збігаються до точного розв'язку диференціально-функціонального рівняння типу фон Фьорстера, що описує популяцію, залежну від своїх минулих густин станів і від загального розміру. На бічній границі ми накладаємо умову відновлення.

Ключові слова: ітеративний метод, диференціальний функціонал, оператор Гейла

PACS: 87.10.+e, 82.39.-k, 82.39.Rk 